\title{
ADESÃO ÀS BOAS PRÁTICAS OBSTÉTRICAS: CONSTRUÇÃO DA ASSISTÊNCIA QUALIFICADA EM MATERNIDADES-ESCOLAS
}

\author{
ADHERENCE TO GOOD OBSTETRIC PRACTICES: \\ BUILDING QUALIFIED ASSISTANCE IN \\ MATERNITY SCHOOLS
}

\section{ADHESIÓN A LAS BUENAS PRÁCTICAS OBSTÉTRICAS: CREACIÓN DE ASISTENCIA CUALIFICADA EN LAS MATERNIDADES ESCUELAS}

\author{
Lahys Firmino Silva ${ }^{1}$ \\ Maria Elisângela Torres de Lima Sanches ${ }^{2}$ \\ Amuzza Aylla Pereira Santos ${ }^{3}$ \\ Julio Cesar Silva Oliveira ${ }^{4}$ \\ Deborah Moura Novaes Acioli ${ }^{5}$ \\ José Augustinho Mendes Santos
}

Como citar este artigo: Silva LF, Sanches METL, Santos AAP, Oliveira JCS, Aciole DMN, Santos JAM. Adesão às boas práticas obstétricas: construção da assistência qualificada em maternidades-escolas. Rev baiana enferm. 2021;35:e37891.

Objetivo: analisar a frequência da realização das boas práticas obstétricas em maternidades-escolas. Método: trata-se de estudo descritivo, retrospectivo e documental, de abordagem quantitativa, que analisou 428 prontuários. Resultados: 90,91\% das parturientes possuíam acompanhante, $81,82 \%$ alimentou-se, $82,50 \%$ pariu em posição verticalizada e $83,12 \%$ teve contato pele a pele. O clampeamento precoce apresentou-se em $28,90 \%$, e $44,17 \%$ usaram ocitocina sintética. Foram observados a amniotomia $(15,00 \%)$, o uso do partograma $(37,50 \%)$ e aplicação de métodos não farmacológicos para alívio da dor (43,18\%). Conclusão: nas maternidades-escolas analisadas, a frequência da realização das boas práticas obstétricas ocorria de forma mais criteriosa em alguns casos, mas ainda seria necessária adequação da assistência.

Descritores: Parto Humanizado. Tocologia. Enfermagem Obstétrica.

Objective: to analyze the frequency of good obstetric practices in maternity schools. Method: this is a descriptive, retrospective and documental study of a quantitative approach, which analyzed 428 medical records. Results: $90.91 \%$ of the women in labor had a companion, $81.82 \%$ had been fed, $82.50 \%$ had childbirth in vertical position and $83.12 \%$ had skin to skin contact. Early clamping was present in $28.90 \%$, and $44.17 \%$ used synthetic oxytocin. It was observed the amniotomy (15.00\%), the use of the partograph (37.50\%) and the application of non-pharmacological

Enfermeira. Pesquisadora Independente. Maceió, Alagoas, Brasil. http://orcid.org/0000-000 I-8987-3825.

Enfermeira. Mestre em Enfermagem. Docente da Universidade Federal de Alagoas. Maceió, Alagoas, Brasil. http://orcid.org/0000-000I-8987-3825.

Enfermeira. Doutora em Ciências da Saúde. Docente da Universidade Federal de Alagoas. Maceió, Alagoas, Brasil. amuzza.santos@gmail.com. http://orcid.org/0000$0001-6299-7190$.

Enfermeiro. Universidade Federal de Alagoas. Maceió, Alagoas, Brasil. http://orcid.org/0000-0003-2267-90I0

Enfermeira. Universidade Federal de Alagoas. Maceió, Alagoas, Brasil. http://orcid.org/0000-0002-3295-8606.

Enfermeiro. Universidade Federal de Alagoas. Maceió, Alagoas, Brasil. http://orcid.org/0000-0002-I570-4I02. 
methods for pain relief (43.18\%). Conclusion: in the maternity schools analyzed, the frequency of good obstetric practices occurred more criteriously in some cases, but it would still be necessary to adjust the assistance.

Descriptors: Humanized Childbirth. Tocology. Obstetric Nursing.

Objetivo: analizar la frecuencia de las buenas prácticas obstétricas en las maternidades escuelas. Método: es un estudio descriptivo, retrospectivo y documental, de enfoque cuantitativo, que analizó 428 registros médicos. Resultados: 90,91\% de las mujeres en parto tuvieron acompañante, 81,82\% fueron alimentadas, 82,50\% tuvieron un parto en posición vertical, y 83,12\% tuvieron contacto piel a piel. El pinzamiento temprano fue del 28,90\%, y el 44,17\% usó oxitocina sintética. Se observó la amniotomía (15,00\%), el uso de la partografía (37,50\%) y la aplicación de métodos no farmacológicos para el alivio del dolor (43,18\%). Conclusión: en las maternidades escuelas analizadas, la frecuencia de las buenas prácticas obstétricas era más juiciosa en algunos casos, pero aun así sería necesario ajustar la atención.

Descriptores: Parto Humanizado. Tocología. Enfermería Obstétrica.

\section{Introdução}

A assistência ao parto, até o final do século XIX, era realizada por parteiras em atendimento domiciliar. O processo de parir fora da residência era algo que acontecia em situações extremas, assim como a presença do profissional médico na cena do parto, que se dava apenas em casos complicados. Esse cenário foi se modificando à medida que a profissão médica foi se consolidando e sendo reconhecida, tornando a mulher e sua vida reprodutiva objeto de estudo nas faculdades de medicina ${ }^{(1)}$.

No Brasil, a maternidade passou a ser interesse do Estado a partir da década de 1930. Nesse período, o ambiente de assistência ao parto passou por uma transição, migrando do domić́lio para o hospital. Além disso, os hábitos maternos começaram a se modificar, deixando de ser intrínsecos ao ser feminino, para tornar-se moldados e orientados por profissionais durante as consultas periódicas ${ }^{(2)}$.

O modelo de atenção à saúde vigente inclui a assistência ao parto e nascimento como um evento médico. Nele a mulher é considerada uma máquina complexa, fragmentada e incapaz de compreender suas necessidades e de tomar decisões sobre sua saúde. Sendo assim, o parto desta mulher defeituosa é realizado pelo profissional médico que, para corrigir as imperfeições, utiliza tecnologias. Estas são empregadas sem a devida avaliação de sua real necessidade, eficácia e segurança ${ }^{(3)}$.
A Organização Mundial de Saúde (OMS) compreende que o parto é um evento fisiológico e, por esta razão, precisa de cuidados e, na maioria das vezes, não necessita de intervenções. Assim, as práticas durante a assistência ao parto e nascimento devem estar de acordo com evidências científicas que unam arte e ciência, proporcionando um nascimento humano e seguro para a mãe e a criança $^{(3)}$. Essas práticas são classificadas em quatro categorias (A, B, C e D), de acordo com os estudos produzidos acerca de cada procedimento $^{(4)}$.

Os hospitais de ensino, conforme Portaria Interministerial MEC/MS no 1.000 , de 2004, são unidades nas quais atividades práticas curriculares são desenvolvidas por acadêmicos de cursos gerais ou especializados da área da saúde pertencentes ou conveniados às instituições de ensino superior. As práticas, nesse cenário, contribuem significativamente para a construção do perfil profissional, visto que, nesse ambiente, há o encontro e a troca de experiência entre estudantes e profissionais ${ }^{(5)}$, garantindo, aos estudantes, a oportunidade de acompanharem o desenvolvimento do trabalho de parto e parto, e de prestarem uma assistência baseada nas evidências científicas produzidas. Nesse contexto, habitualmente, são respeitadas as rotinas da instituição e os conhecimentos do profissional assistente. Entretanto, a assistência ao parto no Brasil, 
inclusive nos hospitais de ensino, tem-se mostrado tecnocrática e centrada na medicalização ${ }^{(1)}$.

Dessa forma, conhecer o cenário da assistência obstétrica, no que se refere às práticas baseadas em evidências científicas, principalmente em maternidades-escolas, instituições formadoras de novos profissionais, pode contribuir para a construção de um modelo de ensino em conformidade com as diretrizes da Rede Cegonha, como recomenda o Ministério da Saúde (MS).

Diante do exposto, o presente estudo tem como objetivo analisar a frequência da realização das boas práticas obstétricas em maternidades-escolas.

\section{Método}

Estudo descritivo, retrospectivo e documental, de abordagem quantitativa. A pesquisa foi realizada em duas maternidades-escolas de alto risco, uma federal e a outra estadual, em uma capital do Nordeste brasileiro. Para a composição da amostra, foi realizado um levantamento documental arquivado, por meio de prontuários, fichas de atendimento obstétrico e livros de registro utilizados durante o acolhimento da gestante até o parto no período de janeiro a junho de $2016^{(6)}$. No total foram analisados 428 prontuários, de março a abril de 2017 com uma média de 214 prontuários por mês.

Para a coleta de dados, elaborou-se um formulário estruturado com dados de identificação, para caracterizar a amostra e os dados específicos sobre o atendimento às parturientes. Os dados foram coletados após aprovação do projeto pelo Comitê de Ética e Pesquisa da Universidade Federal de Alagoas, processo no 63007816.5.0000.5013. Foram incluídos no estudo prontuários com informações sobre a assistência prestada às mulheres que apresentavam mais de 34 semanas de gestação e pariram durante o primeiro semestre de 2016 nas maternidades-escolas. Foram excluídos os prontuários identificados como gestação anembrionária ou outra malformação fetal.

As variáveis utilizadas no estudo foram: idade, gestações e paridade, idade gestacional, presença do acompanhante, utilização do partograma, utilização da episiotomia, manobras, realização da amniotomia, uso de ocitocina, Manobra de Kristeller, métodos não farmacológicos para alívio da dor, posição do parto, dieta prescrita, contato pele a pele, clampeamento do cordão umbilical, peso fetal, aleitamento materno na primeira hora de vida.

Para a organização, tabulação e análise dos dados estatísticos descritivos, utilizou-se o programa IBM SPSS Statistic. A análise descritiva ocorreu com base na frequência absoluta (n) e percentual (F\%) e os resultados foram apresentados de forma descritiva, por meio de tabelas.

\section{Resultados}

Observou-se que a idade das 428 parturientes atendidas nas duas unidades variou entre 13 e 45 anos, sendo a idade média de 24,09 anos com desvio padrão de \pm 7,38 na Federal e 23,73 anos com desvio padrão de \pm 7,01 na Estadual. A idade que apareceu com maior frequência foi 19 anos, com 9,74\%, na Federal e 22 anos, com 8,33\%, na Estadual. Com relação à paridade, na Federal, a maioria apresentou entre 1 a 3 partos; na Estadual, a maioria era nulípara. Ao analisar a idade gestacional em ambas as maternidades-escolas, a maioria estava entre 37 a 39 semanas e 6 dias.

No que se refere às práticas classificadas pela OMS na Categoria A de recomendações, obteve-se que a maioria absoluta possuiu acompanhantes (90,91\% e 86,67\%). Entretanto, a utilização de métodos não farmacológicos para o alívio da dor esteve presente para a minoria $(43,18 \%$ e $12,50 \%)$, como mostra a Tabela 1. 
Tabela 1 - Frequência das práticas demonstrativamente úteis e que devem ser estimuladas, Categoria A, em maternidades-escolas de alta complexidade. Alagoas, Brasil - 2017

\begin{tabular}{l|c|c|c|c}
\hline \multirow{2}{*}{ Variáveis } & \multicolumn{2}{c|}{ Federal } & \multicolumn{2}{c}{ Estadual } \\
\cline { 2 - 5 } & $\begin{array}{c}\text { Amostra } \\
\text { (n= 308) }\end{array}$ & $\mathbf{\%}$ & $\begin{array}{c}\text { Amostra } \\
\text { (n= 120) }\end{array}$ & \% \\
\hline Presença de acompanhante & 280 & 90,91 & 104 & 86,67 \\
Prescrição de hidratação e alimentação por via oral & 252 & 81,82 & 46 & 38,33 \\
Posição verticalizada durante o parto & 192 & 62,34 & 99 & 82,50 \\
Aleitamento materno na primeira hora de vida & 206 & 66,88 & 40 & 33,33 \\
Contato pele a pele & 256 & 83,12 & 95 & 79,17 \\
Utilização de métodos não farmacológicos para o & 133 & 43,18 & 15 & 12,50 \\
alívio da dor & & & & \\
Partograma & - & - & 45 & 37,50 \\
\hline
\end{tabular}

Fonte: Elaboração própria.

Nota: Sinal convencional utilizado:

- Dado numérico igual a zero não resultante de arredondamento.

A maioria expressiva das mulheres foi alimentada e hidratada durante o trabalho de parto e parto, conforme descrito em uma das unidades, ao passo que, na outra instituição, mais da metade das mulheres foi mantida em jejum.

Tabela 2 - Frequência de práticas claramente prejudiciais e que devem ser abolidas, Categorias B, C e D, em maternidades-escolas de alta complexidade. Alagoas, Brasil - 2017

\begin{tabular}{l|c|c|c|c}
\hline \multirow{2}{*}{ Variáveis } & \multicolumn{2}{|c|}{ Federal } & \multicolumn{2}{c}{ Estadual } \\
\cline { 2 - 5 } & $\begin{array}{c}\text { Amostra } \\
(\mathbf{n = 3 0 8})\end{array}$ & $\mathbf{\%}$ & $\begin{array}{c}\text { Amostra } \\
\text { (n= 120) }\end{array}$ & $\mathbf{\%}$ \\
\hline Litotomia & 104 & 33,77 & 14 & 11,67 \\
Infusão de Ocitocina & 93 & 30,20 & 53 & 44,17 \\
Clampeamento precoce do cordão umbilical & 89 & 28,90 & 6 & 5,00 \\
Amniotomia & 7 & 2,27 & 18 & 15,00 \\
Episiotomia & 58 & 18,83 & 25 & 20,83 \\
Manobra de Kristeller & - & - & 2 & 1,67 \\
\hline
\end{tabular}

Fonte: Elaboração própria.

Nota: Sinal convencional utilizado:

- Dado numérico igual a zero não resultante de arredondamento.

Obteve-se baixa frequência de realização de amniotomia, prática classificada na categoria $\mathrm{C}$ pela OMS, porém 97,40\% dos prontuários da Federal não continham informações referentes a esta prática.

Percebe-se uma grande omissão nos registros do profissional assistente, visto que 17,99\% $(n=77)$ de todos os partos assistidos nas duas unidades $(n=428)$ não possuíam anotações referentes à integridade perineal.

\section{Discussão}

O estudo evidenciou que as idades das parturientes atendidas nas duas maternidades estavam fora da faixa etária considerada como fator gerador de risco pelo MS, pois a minoria possuía idade inferior ou igual a 15 anos e superior a 35 anos. Entretanto, é válido ressaltar que a idade, por si só, não representa risco, pois precisa estar associada a outros fatores que, somados, possam ser a causa de agravos à gestação e, consequentemente, resultar em danos para a saúde materno-infantil, elevando, assim, a morbimortalidade materno-fetal ${ }^{(7-8)}$. Dentre os fatores que caracterizam a população estudada, observou-se que, apesar de não aparecer com maior frequência em alguns estudos, a nuliparidade mostrou-se significativa na amostra estudada. Este é também 
um fator gerador de risco gestacional, de acordo com o MS. Além disso, metade da população possuía idade gestacional correspondente ao termo, fato contraditório em relação aos critérios de elegibilidade de risco ${ }^{(6,9)}$.

As boas práticas obstétricas são classificadas pela OMS em categorias que ajudam na assistência qualificada às parturientes e baseiam-se em experiências exitosas. No decorrer dos anos, os estudos realizados foram construindo evidências para reforçar as recomendações gerais sobre a assistência prestada à parturiente, fortalecendo, assim, as Diretrizes Nacionais de Assistência ao Parto Normal. Estas afirmam que as boas práticas obstétricas servem também para partos que apresentam risco gestacional $^{(6,10)}$.

Dentre essas práticas têm-se a presença do acompanhante, reconhecida como direito previsto na Lei Federal no $11.108 / 2005$. Tal prática está dentro da categoria A, isto é, são indubitavelmente benéficas e devem ser estimuladas, uma vez que o apoio físico e emocional contínuo promove melhores desfechos do parto, principalmente quando são provenientes de pessoas da convivência da gestante e transmitem-lhe confiança e segurança. Ao avaliar sua aplicação nas maternidades estudadas, obteve-se que a grande maioria das mulheres teve seu direito respeitado, quando as maternidades permitiram ao acompanhante participar de toda a assistência prestada durante o trabalho de parto e parto. A segurança transmitida pelo acompanhante, no momento do parto, é de extrema importância para o prosseguimento do processo. Assim, os profissionais de saúde, além de assegurarem às mulheres e a seus familiares esse direito, devem estimular não somente a presença, mas, principalmente, a participação e o envolvimento do familiar. Para isso, pode-se instruí-los sobre técnicas de relaxamento, respiração e suporte físico e emocional, proporcionando-lhes privacidade e um ambiente acolhedor ${ }^{(11)}$.

Outra prática que deve ser incentivada é o estímulo à hidratação e alimentação oral durante todo o trabalho de parto e parto, pois as mulheres necessitam de um aporte energético elevado para que consigam um bom desenvolvimento do processo parturitivo, visto que, de acordo com as evidências científicas, a dieta leve e a ingestão de líquidos calóricos aumentam os níveis de glicose e insulina e promovem a diminuição da produção de corpos cetônicos pela fadiga muscular. Ao analisar os resultados obtidos no estudo, verificou-se, entre as duas instituições estudadas, que os resultados foram contrários. A instituição estadual pratica a alimentação das parturientes, e a instituição federal impõe o jejum a mais da metade delas. Ainda que as parturientes atendidas nas instituições em estudo apresentem alto risco gestacional, a ingestão hídrica, assim como a ingesta de alimentos leves não está contraindicada, visto que não há evidências científicas fortes que comprovem a necessidade da dieta zero para este grupo ${ }^{(12)}$.

Além das recomendações anteriormente descritas, é incentivado também o uso do partograma, por tratar-se de um instrumento gráfico no qual são registradas as condições maternas e fetais, permitindo o acompanhamento e a avaliação do trabalho de parto. No partograma, devem conter informações indicadas pela OMS, como a dilatação cervical, a dinâmica uterina, a altura da apresentação, a frequência cardíaca fetal, assim como os sinais vitais da parturiente. Esses registros ajudam a equipe a direcionar intervenções indispensáveis às necessidades clínicas que a parturiente possa apresentar durante o estágio de trabalho de parto, diminuindo o risco de mortalidade materno-fetal ${ }^{(13)}$.

Entretanto, neste estudo não foram encontrados dados referentes ao preenchimento do partograma em uma das unidades; na outra, a frequência de uso foi baixa. O não registro dos dados referentes ao trabalho de parto no partograma impossibilita analisar se houve ou não necessidade das intervenções realizadas no grupo estudado, visto que o preenchimento correto desse documento subsidia o poder de decisão do profissional assistente.

Além dos fatores técnicos, devem ser levados em consideração elementos subjetivos, como, por exemplo, a dor. Ela é considerada como o principal componente do parto vaginal e é responsável pelo sentimento de medo demonstrado 
pelas gestantes. Entretanto, a dor durante o trabalho de parto é fisiológica e importante, pois desencadeia a liberação de endorfinas, hormônios que ajudam na realização da finalização do trabalho de parto, proporcionando o prazer e a satisfação. Dessa forma, a assistência obstétrica não deve ser centrada em promover ausência de dor, e sim a utilização de métodos não farmacológicos para seu alívio durante o trabalho de parto e parto ${ }^{(12)}$.

Os resultados deste estudo demonstram que menos da metade das parturientes atendidas tiveram acesso a esses métodos, embora as maternidades possuam meios para realizá-los. Em uma das maternidades, estão disponíveis materiais e ambiente propício para a musicoterapia, aromaterapia, rebozos, escalda pés; em ambas estão disponíveis massagem, uso de bola suíça, penumbra e banho morno. Esses recursos podem proporcionar à parturiente momentos de alívio da dor e acolhimento durante sua estadia na sala de parto. Além disso, a verticalização do parto tem sido estimulada em virtude do favorecimento gravitacional ao mecanismo de parto, para gerar maior conforto e satisfação à mulher quanto à parição. A litotomia ou posição supina, por sua vez, é comprovadamente prejudicial, fazendo-se necessária sua abolição e substituição por posições como semissentada, sentada, de cócoras, entre outras $^{(13)}$. Neste estudo, viu-se que as parturientes, em sua maioria, pariram em posição verticalizada, porém um significativo número de partos foi realizado em litotomia.

É importante observar que impor a posição que a mulher deve parir, seja ela verticalizada ou não, é contraindicado, pois, nesse momento, ela deve sentir conforto e segurança para o período expulsivo. A adoção de posições mais verticais é inerente ao ser humano, entretanto, em virtude da cultura da proibição de movimentação e imposição do decúbito dorsal, as mulheres adotam tal posição espontaneamente. Nesse contexto, o profissional de saúde assistente deve apresentar, orientar e estimular a adesão a posições verticalizadas $^{(14)}$.

Outra ação que também é incentivada é o contato pele a pele. Este contato consiste em colocar o bebê sem roupa, de bruços, sobre o tórax ou abdômen materno, cobrindo-o para mantê-lo aquecido, promovendo, assim, melhor adaptação do recém-nascido à vida extrauterina ${ }^{(14)}$. Além disso, estudos têm comprovado que o contato precoce facilita a promoção da amamentação e a construção de vínculo na primeira hora do pós-parto. Essas práticas são estabelecidas como rotina na maioria das maternidades. Entretanto, muitas vezes, esse momento é curto e não respeita as normas preconizadas pela Iniciativa Hospital Amigo da Criança ${ }^{(15)}$.

Este estudo evidenciou que a maior parte dos recém-nascidos foi colocada em contato pele a pele, porém o percentual significativo referente ao clampeamento precoce do cordão umbilical (prática classificada na categoria $\mathrm{C}$ de recomendações da OMS) levanta a hipótese de que este primeiro contato entre mãe e filho não está em conformidade com o recomendado, fator que pode influenciar negativamente no estabelecimento de vínculo entre o binômio mãe-bebê ${ }^{(15)}$.

Quanto à infusão endovenosa de ocitocina sintética durante o trabalho de parto, cerca de um terço da população em uma das unidades, e quase metade das mulheres na outra instituição foi submetida a esta prática. Estes números, apesar de representarem a minoria, são significativos e devem ser levados em consideração, visto que o uso rotineiro de uterotônicos é contraindicado pela OMS e pelo Ministério de Saúde. O uso da ocitocina no trabalho de parto deve ser baseado em critérios clínicos. Ela não deve ser empregada para acelerar o parto, entretanto sua aplicação com esta finalidade ainda é muito frequente, como mostram alguns estudos ${ }^{(16-17)}$. A restrição dessa prática decorre do efeito deletério na parturiente, visto que o medicamento aumenta a frequência e a intensidade das contrações uterinas, o que implica em maior desconforto e risco de rotura uterina e sofrimento fetal ${ }^{(12)}$.

Diante do exposto, percebe-se que os profissionais das unidades em estudo podem estar utilizando esse método para reduzir o tempo de trabalho de parto. Entretanto, sob a ótica do cuidado humanizado, essa rotina é potencialmente prejudicial, não apenas levando em consideração 
a intervenção sobre o processo fisiológico, mas também por impor à mulher um aumento significativo da dor, o que pode provocar danos emocionais, principalmente no que se refere à satisfação natural de gerar e parir um filho ${ }^{(15)}$.

Uma das primeiras intervenções realizadas logo após o nascimento é o clampeamento precoce do cordão umbilical. Este é definido como o pinçamento e secção do cordão antes de um minuto após o nascimento do bebê, sendo o tardio, ou oportuno, aquele que se dá entre um e cinco minutos, ou somente após a cessação de sua pulsação. Sua execução antecipada é contraindicada, visto que seu uso deve ser cauteloso, isto é, apenas com indicação clínica estabelecida, como, por exemplo, a prevenção da transmissão vertical do $\mathrm{HIV}^{(18)}$.

O presente estudo mostra que essa prática apresenta-se de maneiras diferentes nas duas instituições. Em uma delas, na maior parte dos partos assistidos foi realizado o clampeamento oportuno do cordão umbilical, indicador positivo de qualidade da assistência prestada. Seus benefícios para o recém-nascido têm sido comprovados, como maior aporte de ferro na primeira infância e contribuição para melhores êxitos nos casos em que é necessário o emprego de manobras de reanimação neonatal ${ }^{(9,16)}$. Em contrapartida, a outra instituição registrou o clampeamento oportuno em menos de um quarto da população em estudo, fato este que pode futuramente trazer alguns transtornos ao recém-nascido ${ }^{(15,18)}$.

Outro procedimento muito recorrente na assistência ao parto é a amniotomia, a qual consiste no rompimento artificial das membranas ovulares, como meio de acelerar o trabalho de parto, mas também para avaliar o aspecto do líquido amniótico em situações que exigem atenção de maior complexidade. Seu uso rotineiro é contraindicado, visto que implica em riscos, como o prolapso de cordão umbilical e ameaça para infecção materna e neonatal ${ }^{(8)}$. Esse procedimento não se demonstrou uma prática presente neste estudo, porém este dado pode não corresponder à realidade em uma das instituições, visto que os registros quanto à realização ou não dessa prática foram omitidos na maioria absoluta dos casos. Por outro lado, uma das instituições apresenta baixos índices de aplicação da técnica.

Dentre as intervenções realizadas indiscriminadamente está a episiotomia, incisão cirúrgica realizada no períneo com intenção de ampliar o canal vaginal, uma das práticas mais utilizadas em todo o mundo. Seu uso indiscriminado está associado ao aumento da perda sanguínea, dor perineal e maior risco de laceração perineal grave (Grau III e IV), não apresentando benefícios maternos claros. Esse procedimento foi realizado em menos de um quarto da população em estudo das duas instituições, o que indica que a prática ainda está distante do percentual máximo recomendado pela $\mathrm{OMS}^{(16)}$.

Dentre as práticas mais comuns no cenário obstétrico brasileiro, encontra-se a manobra de Kristeller, a qual consiste em comprimir o fundo uterino, objetivando abreviar o período expulsivo. Entretanto, seu registro oficial é inexistente na maior parte dos hospitais, como observado no presente estudo. Em uma das unidades, por exemplo, não foram encontrados registros relacionados ao uso da técnica, considerada como potencialmente prejudicial para mãe e bebê. Sendo assim, é necessária sua extinção das salas de parto e centros obstétricos ${ }^{(17)}$. É imprescindível salientar a importância do envolvimento das instituições assistenciais, principalmente as formadoras de novos profissionais, no combate às práticas potencialmente prejudiciais, especialmente aquelas que devem ser extintas, como a manobra de Kristeller.

É válido ressaltar que o parto normal não está contraindicado para gestantes de alto risco. A sua elegibilidade depende não somente da trajetória percorrida pela gestante durante todo o pré-natal, mas, principalmente, da situação de saúde da gestante no momento do trabalho de parto e parto, assim como da condução adotada pela equipe que prestará assistência à parturiente nesse momento tão importante e único para sua vida ${ }^{(1)}$.

Esta pesquisa apresentou como limitação a omissão de dados importantes nos registros dos profissionais assistentes. Além disso, observou-se uma repetida contradição entre as informações contidas na evolução médica e nas 
de enfermagem, principalmente relacionadas às práticas contraindicadas pelo MS. Outro fato relacionado ao estudo foi a perda de alguns dados, uma vez que, em uma das instituições, o acesso ao prontuário físico foi dificultado. Sendo assim, a pesquisa foi realizada no prontuário eletrônico, que não continha todas as informações.

\section{Conclusão}

Este estudo evidenciou que o cenário obstétrico analisado está passando por transformações, haja vista os bons resultados referentes a algumas das práticas analisadas. Constatou-se que as parturientes tiveram seu direito ao acompanhante preservado, entretanto, ainda passavam pelo processo de parturição em jejum. Além disso, uma grande parte dessas mulheres teve acesso a posições verticalizadas e ao contato imediato com seu filho.

Em contrapartida, a maioria não teve acesso aos métodos não farmacológicos para o alívio da dor, prática incentivada e definida como benéfica pela OMS e pelos programas ministeriais de incentivo à atenção humanizada. Ademais, as intervenções no processo de trabalho de parto e parto, como o uso de ocitocina endovenosa e a episiotomia, não se mostraram presentes na maioria dos atendimentos realizados, porém ainda não estão em consonância com o preconizado.

É importante enfatizar a necessidade de adequação das maternidades-escolas vinculadas no estudo, já que são formadoras de profissionais desde a graduação. Desta forma, é relevante a realização de outros estudos, de maneira que seja possível analisar a evolução e o ajuste dessas instituições, proporcionando um cenário obstétrico favorável e formador de qualidade.

\section{Colaborações:}

1 - concepção, projeto, análise e interpretação dos dados: Lahys Firmino Silva, Maria Elisângela Torres de Lima Sanches e Amuzza Aylla Pereira Santos;
2 - redação do artigo e revisão crítica relevante do conteúdo intelectual: Lahys Firmino Silva, Maria Elisângela Torres de Lima Sanches, Amuzza Aylla Pereira Santos, Julio Cesar Silva Oliveira, Deborah Moura Novaes Acioli e José Augustinho Mendes Santos;

3 - aprovação final da versão a ser publicada: Lahys Firmino Silva e Amuzza Aylla Pereira Santos.

\section{Referências}

1. Duarte SJH, Machado RM. Competencias esenciales de la formación en obstetricia. Rev Panam Salud Pública [Internet]. 2016 nov [cited 2020 Sep 7];40(5):382-7. Available from: https://www. scielosp.org/scielo.php?script=sci_arttext\&nrm= is $o \& \operatorname{lng}=\mathrm{pt} \& \mathrm{t} \ln \mathrm{g}=\mathrm{pt} \& \mathrm{pid}=\mathrm{S} 1020-49892016$ 001100382

2. Ramos WMA, Aguiar BGC, Conrad D, Pinto CB, Mussumeci PA. Contribuição da enfermeira obstétrica nas boas práticas da assistência ao parto e nascimento. Rev Fund Care Online. 2018 jan/mar;10(1):173-9. DOI: http://dx.doi.org/ 10.9789/2175-5361.2018.v10i1.173-179

3. Brasil. Ministério da Saúde. Humanização do parto e do nascimento [Internet]. Brasília (DF); 2014. (Cadernos Humaniza SUS; v. 4). [cited 2020 Sep 10]. Available from: https:// portaldeboaspraticas.iff.fiocruz.br/biblioteca/ humanizacao-do-parto-e-do-nascimento/

4. Organização Mundial da Saúde. Tecnologia apropriada para partos e nascimentos. Recomendações da Organização Mundial de Saúde. Maternidade Segura. Assistência ao parto normal: um guia prático [Internet]. Genebra; 1996 [cited 2020 Sep 2]. Available from: https://saude. mppr.mp.br/arquivos/File/kit_atencao_perinatal/ manuais/assistencia_ao_parto_normal_2009.pdf

5. Brasil. Ministério da Saúde. Ministério da Educação. Portaria Interministerial $\mathrm{n}^{-} \mathbf{1 . 0 0 0}$, de 15 de abril de 2004. Certifica como Hospital de Ensino as Instituições Hospitalares que servirem de campo para prática de atividades curriculares na área da saúde, sejam Hospitais Gerais e, ou Especializados, de propriedade de Instituição de Ensino Superior, pública ou privada, ou ainda, formalmente conveniada com Instituições de Ensino Superior [Internet]. Brasília (DF); 2004. [cited 2020 Aug 25]. Available from: https://www. 
diariodasleis.com.br/busca/exibelink.php?num link=1-92-31-2004-04-15-1000

6. Vieira MJO, Santos AAP, Silva JMO, Sanches METL. Assistência de enfermagem obstétrica baseada em boas práticas: do acolhimento ao parto. Rev Eletr Enf. 2016;18:e1166. DOI: http://dx.doi. org/10.5216/ree.v18.36714

7. Rabelo LR, Oliveira DL. Percepções de enfermeiras obstétricas sobre sua competência na atenção ao parto normal hospitalar. Rev Esc Enferm USP. 2010 mar;44(1):213-20. DOI: https://doi. org/10.1590/S0080-62342010000100030

8. Klerk HW, Boere E, Lunsen RH, Bakker JJH. Women's experiences with vaginal examinations during labor in the Netherlands. J Psychosom Obstet Gynecol. 2018 Jun;39(2):90-5. DOI: http:// dx.doi. org/10.1080/0167482X.2017.1291623

9. Brasil. Ministério da Saúde. Secretaria de Atenção à Saúde. Departamento de Ações Programáticas Estratégicas. Gestação de alto risco: manual técnico. 5 a ed. Brasília (DF); 2012. (Série A. Normas e Manuais Técnicos). [cited 2020 Aug 27]. Available from: http://bvsms.saude.gov.br/bvs/publicacoes/ manual_tecnico_gestacao_alto_risco.pdf

10. Oliveira JC, Paula ACS, Garcia ESGF, Andrade MBT, Leite EPRC. Assistência obstétrica no processo de parto e nascimento. Rev Pesqui Cuid Fundamen. 2018 abr-jun;10(2):450-7. DOI: http://dx.doi. org/10.9789/2175-5361.2018.v10i2.450-457

11. Rodrigues DP, Alves VH, Penna LHG, Pereira AV, Branco MBLR, Souza RMP. O descumprimento da lei do acompanhante como agravo à saúde obstétrica. Texto contexto - enferm. 2017;26(3):e5570015. DOI: http://dx.doi.org/10. 1590/0104-07072017005570015

12. Porto AMF, Amorim MMR, Souza ASR. Assistência ao primeiro período do trabalho de parto baseada em evidências. Femina [Internet] 2010 out [cited 2020 Sep 7];38(10):527-37. Available from: http://bhpelopartonormal.pbh.gov. br/estudos_cientificos/arquivos/artigo_femina_ assistencia_ao_parto_parte_I.pdf

13. Freire HSS, Campos FC, Castro RCMB, Costa CC, Mesquita VJ, Viana RAA. Parto normal assistido por enfermeira: experiência e satisfação de puérperas. Rev Enferm UFPE. 2017 jun;11(6):2357-67. DOI: 10.5205/reuol.10827-96111-1-ED.1106201714

14. Lotto CR, Linhares MBM. Contato "Pele a Pele" na Prevenção de Dor em Bebês Prematuros: Revisão Sistemática da Literatura. Trends Psychol. 2018;26(4):1699-713. DOI: http://dx.doi. org/10.9788/tp2018.4-01pt

15. Andrade PON, Silva JQP, Diniz CMM, Caminha MFC. Fatores associados à violência obstétrica na assistência ao parto vaginal em uma maternidade de alta complexidade em Recife, Pernambuco. Rev Bras Saúde Mater Infant. 2016 jan/ mar;16(1):29-37. DOI: https://doi.org/10.1590/ 1806-93042016000100004

16. Pompeu KC, Scarton J, Cremonese L, Flores RG, Landerdahi MC, Ressel LB. Prática da episiotomia no parto: desafios para a enfermagem. Rev Enferm Cent-Oeste Min. 2017;7:e1142. DOI: 10.19175/ recom.v7i0.1142

17. Sousa AMM, Souza KV, Rezende EM, Martins EF, Campos D, Lansky S. Práticas na assistência ao parto em maternidades com inserção de enfermeiras obstétricas, em Belo Horizonte, Minas Gerais. Esc Anna Nery. 2016;20(2):324-31. DOI: https://doi.org/10.5935/1414-8145.20160044

18. Sobieray NLEC, Neves IS, Skrobot T. Relação entre o tempo de clampeamento do cordão umbilical e incidência de Icterícia Neonatal e níveis de hematócrito em recém-nascidos a termo saudáveis. Arq Med Hosp Fac Cienc Med Santa Casa São Paulo. 2019 maio/ago;64(2):88-92. DOI: https:// doi.org/10.26432/1809-3019.2019.64.2.088

Recebido: 14 de julho de 2020 Aprovado: 29 de setembro de 2020 Publicado: 1 de dezembro de 2020

A Revista Baiana de Enfermagem utiliza a Licença Creative Commons - Atribuição-NãoComercial 4.0 Internacional. https://creativecommons.org/licenses/by-nc/4.0/ Este artigo é de acesso aberto distribuído sob os termos da Licença Creative Commons (CC BY-NC). Esta licença permite que outros remixem, adaptem e criem a partir do seu trabalho para fins não comerciais. Embora os novos trabalhos tenham de lhe atribuir o devido crédito e não possam ser usados para fins comerciais, os usuários não têm de licenciar esses trabalhos derivados sob os mesmos termos. 